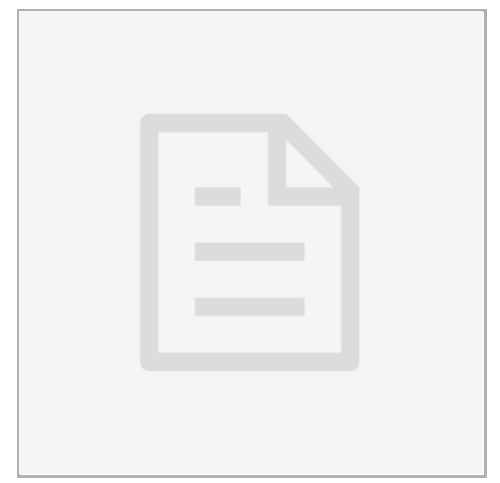

JUN 08, 2018

\section{open $\boldsymbol{O}$ access}

\section{DOI:}

dx.doi.org/10.17504/protocol s.io.qq9dvz6

Protocol Citation: Xin Liu 2018. An analytical pipeline of assembly and annotation of the Betta splendens genome.. protocols.io

https://dx.doi.org/10.17504/p rotocols. io. qq9dvz6

\section{License: This is an open} access protocol distributed under the terms of the Creative Commons Attribution License, which permits unrestricted use, distribution, and reproduction in any medium, provided the original author and source are credited

\section{Protocol status: Working}

Created: Jun 06, 2018

Last Modified: Jun 08, 2018

PROTOCOL integer ID:

12801

\section{(3) An analytical pipeline of assembly and annotation of the Betta splendens genome.}

$\rightarrow$ In 2 collections

Xin Liu ${ }^{1}$

${ }^{1} \mathrm{BGI}-$ Shenzhen

\section{GigaScience Press BGI}

Kailong $\mathrm{Ma}$

\section{ABSTRACT}

From here, You can learn about the detail methods of genome assembly and gene annotation of the Betta splendens genome.

\title{
Quality Control
}

1 Get raw sequencing data in Fastq format. Filter the raw sequencing data by using SOAPfilter (version 2.2). 
Using parameters '-i insertsize -y -z - $\mathrm{p}-\mathrm{M} 2$ '

\section{k-mer analysis}

2 Estimate the genome size with k-mer (version 1.0) analysis.

Note

Using parameters ' $\mathrm{k}=17$-t 12 '

\section{Assembly}

3 1. Run SOAPdenovo (version 2.04 ) to assemble the Betta splendens genome.

2. Perform Gapcloser (version 1.12) to further close gaps in our genome obtained in step3.

Note

1-Note: using parameters'pregraph(-K 29 -p 20); contig(-M 2);map(-k 41);scaff(<default>)'

Note

2-Note: using reads from all insert-size libraries

\section{Repeat annotation_de novo}

4 1. Run RepeatModeler(1.0.8) and LTR_FINDER(1.0.6), respectively, to build de novo library based on the input assembled genome sequence.

2. Basing on the library constructed in step 5 as database, run RepeatMasker (version 3.3.0) to find and then classify the repetitive sequences.

Note

1-Note: <default > 
2-Note: using parameters '-nolow -no_is -norna -parallel 1'

\section{Repeat annotation_homolog}

5 Run RepeatMasker and ProteinMask (version 3.3.0) to identify repeats in the genome at DNA and protein level, respectively, by aligning sequences against existing databases, Repbase TE library (Version 17.01) and TE protein database.

Note

Using parameters 'RepeatMasker(-nolow -no_is -norna -parallel 1)ProteinMask(noLowSimple -pvalue 0.0001)'

\section{Gene prediction_de novo}

6 Run Augustus (version 3.0.3) and GlimmerHMM (version 3.0.1) to de novo predict genes in the repeat-masked genome sequences.

Note

Using parameters 'Augustus(--species=zebrafish --uniqueGeneld=true --nolnFrameStop=true --gff3=on --strand=both)GlimmerHMM(-d zebrafish $-\mathrm{f}-\mathrm{g})^{\prime}$

Using parameters '-d zebrafish $-f-g$ '

\section{Gene prediction_homolog}

7 Download protein sequences of homlog species (danio rerio(release-64), gadus morhua(release65), gasterosteus aculeatus(release-64), oryzias latipes(release-64), takifugu rubripes(release64), and tetraodon nigroviridis(release-64)), then align these against our masked genome sequences with BLAT, and then based on the BLAT mapping results, run GeneWise (version 2.2.0 ) to predict genes.

Note

Using parameters '--min_align_coverage 0.3 --max divergence rate 0.3 --extend length for both sides of regions 2000' 


\section{Gene prediction_glean}

8 Integrate genes predicted in step 8-9 to obtain the consensus gene set by using GLEAN.

Note

Filtering with criterion 'overlap cutoff 0.8 and at least one homolog support'

\section{Gene prediction_adding RNA-seq}

9 Perform TopHat (version 2.1.0) with default parameters to align filtered RNA-seq reads against gene set mentioned in Step10, and then use Cufflinks (version 2.2.1) to assemble these transcripts, then use training parameters to predict ORFs, and finally obtain the more intergrity and trusty gene set.

Note

Filtering RNA sequencing data by SOAPnuke with parameters '-I 10 -q 0.5 -n 0.01 -Q 2"

\section{Estimation of completeness}

10 Run BUSCO(version 3.0.1) and map final gene set and genome to actinopterygii reference to assess the completeness.

Note

Using parameters '-e 0.001 -limit 3' 